\title{
Overview of adult congenital heart transplants
}

\author{
Roosevelt Bryant III', ${ }^{1,2}$ David Morales ${ }^{1,2}$ \\ ${ }^{1}$ Division of Cardiovascular Surgery, The Heart Institute, Cincinnati Children's Hospital Medical Center, Cincinnati, OH, USA; ${ }^{2}$ Department of \\ Surgery, The University of Cincinnati College of Medicine, Cincinnati, OH, USA \\ Correspondence to: Roosevelt Bryant III, MD. Associate Professor of Surgery, The University of Cincinnati Medical Center, Surgical Director, Heart \\ Transplant Program, Cincinnati Children’s Hospital Medical Center, 3333 Burnett Avenue, Cincinnati, OH, USA. Email: roosevelt.bryantiii@cchmc.org.
}

\begin{abstract}
Transplantation for adult patients with congenital heart disease (ACHD) is a growing clinical endeavor in the transplant community. Understanding the results and defining potential high-risk patient subsets will allow optimization of patient outcomes. This review summarizes the scope of ACHD transplantation, the mechanisms of late ventricular dysfunction, the ACHD population at risk of developing heart failure, the indications and potential contraindications for transplant, surgical considerations, and post-transplant outcomes. The findings reveal that $3.3 \%$ of adult heart transplants occur in ACHD patients. The potential mechanisms for the development of late ventricular dysfunction include a morphologic systemic right ventricle, altered coronary perfusion, and ventricular noncompaction. The indications for transplant in ACHD patients include systemic ventricular failure refractory medical therapy, Fontan patients failing from chronic passive pulmonary circulation, and progressive cyanosis leading to functional decline. Transplantation in ACHD patients can be quite complex and may require extensive reconstruction of the branch pulmonary arteries, systemic veins, or the aorta. Vasoplegia, bleeding, and graft right ventricular dysfunction can complicate the immediate post-transplant period. The post-transplant operative mortality ranges between 14\% and 39\%. The majority of early mortality occurs in ACHD patients with univentricular congenital heart disease. However, there has been improvement in operative survival in more contemporary studies. In conclusion, the experience with cardiac transplantation for ACHD patients with end-stage heart failure is growing, and high-risk patient subsets have been defined. Significant strides have been made in developing evidence-based guidelines of indications for transplant, and the intraoperative management of complex reconstruction has evolved. With proper patient selection, more aggressive use of mechanical circulatory support, and earlier referral of patients with failing Fontan physiology, outcomes should continue to improve.
\end{abstract}

Keywords: Adult congenital heart disease; Heart transplantation; Mechanical Circulatory support; Surgical outcomes

Submitted Sep 08, 2017. Accepted for publication Jan 10, 2018.

doi: $10.21037 /$ acs.2018.01.01

View this article at: http://dx.doi.org/10.21037/acs.2018.01.01

\section{Introduction}

Transplantation for adult patients with congenital heart disease (ACHD) is a high-risk proposition. Gaining an understanding of which patients will do well with transplant and those who will not remains elusive. There is, however, a growing number of single institution (1-5) and registry based $(6,7)$ studies that attempt to shed light on outcomes. These reports provide the foundation for evidence-based guidelines to counsel patients and families on this growing clinical endeavor in the transplant community. The purpose of this review is to provide the reader with an understanding of the current expectations for ACHD patients being considered for isolated cardiac transplantation. Specifically, we will attempt to frame for the reader: (I) the scope of ACHD transplantation; (II) the mechanisms of late ventricular dysfunction in ACHD patients and the population at risk; (III) the indications and potential contraindications for transplant; (IV) surgical considerations; and (V) post-transplant outcomes. 


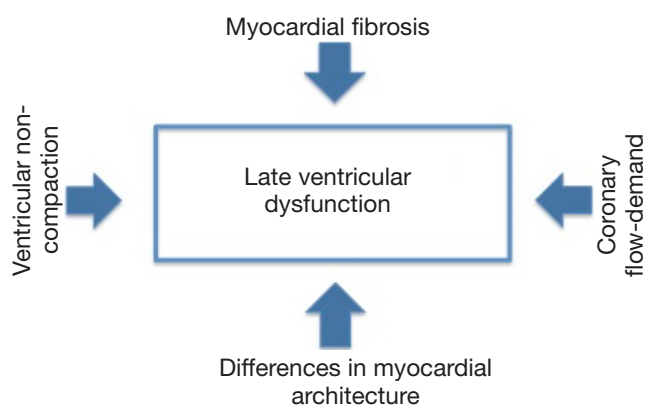

Figure 1 Mechanisms of late ventricular dysfunction in ACHD patients. ACHD, adult patients with congenital heart disease.

\section{The scope of ACHD transplantation}

Current estimates suggest that nearly $90 \%$ of children with repaired congenital heart disease survive into adulthood (8). Residual anatomic issues from previous repair, arrhythmias, and late ventricular dysfunction will put these patients at risk for the development of end stage heart failure $(2,9,10)$. Heart failure accounts for $26 \%$ to $42 \%$ of deaths in ACHD patients $(11,12)$. As a consequence, the transplant community has seen a growing number of referrals and heart transplants in ACHD patients. The most recent registry report from the International Society of Heart and Lung transplantation (ISHLT) indicates that 3.3\% of adult heart transplants occur in patients with congenital heart disease (13). Though this percentage is small relative to nonACHD adult heart transplant recipients, this represents a $40 \%$ increase over previous decades dating back to 1999 (14).

\section{Mechanisms of late ventricular dysfunction in ACHD patients and at-risk populations}

Any patient who underwent repair of a congenital cardiac anomaly as a child, even a relatively simple lesion such as a septal defect, can develop heart failure as an adult. There are, however, certain diagnoses that are particularly prone to the development of late ventricular dysfunction. The potential mechanisms for development of late ventricular dysfunction in repaired congenital heart disease were elucidated in a recent review by Stout and colleagues as a consensus statement of the American Heart Association (15). A systemic right ventricle is a risk factor for late ventricular dysfunction whether it is associated with repaired biventricular or palliated univentricular congenital heart disease (UCHD). Transposition of the great vessels that was repaired by an atrial switch procedure and classic repair of congenitally corrected transposition of the great vessels (ccTGA) are examples of biventricular circulations with a systemic right ventricle (16). The quintessential univentricular heart with a systemic right ventricle is hypoplastic left heart syndrome (HLHS).

The mechanisms for systemic right ventricular dysfunction include architectural changes such as myocardial fiber disarray, myocardial ischemia, and the development of myocardial fibrosis (15) (Figure 1). The normal architecture of the right ventricle may predispose it to late dysfunction in the systemic circulation. In particular, the absence of a middle layer of circular muscle fibers as seen in the morphologic left ventricle may contribute to the right ventricle's response to increased systemic afterload $(15,17)$. Abnormal myocardial fiber orientation has been demonstrated in autopsy specimens of patients with tricuspid atresia (18) and in animal models of HLHS (19). Finally, ventricular noncompaction may also contribute to the development of late ventricular dysfunction. Trabecular compaction forms normal myocardium and contributes to normal contractile function (20). Ventricular noncompaction is more common in patients with congenital heart disease (21). If the process of myocardial compaction fails embryologically, it is lethal to the murine fetus (22). The effectiveness of the systemic right ventricle is also hindered by the tricuspid valve being septophilic. This can lead to significant tricuspid valve insufficiency when the right ventricle changes from a crescent-shaped chamber to a rounded one. Also, it is often noted in HLHS that the septal leaflet of the tricuspid valve can often be restricted in its motion or deficient. The precise interaction of the aforementioned structural changes in the development of late ventricular dysfunction in ACHD patients remains unclear.

Besides structural changes, altered coronary perfusion may also play a role in the development of systemic right ventricular dysfunction. The usual coronary branching and perfusion may be inadequate in patients with a systemic right ventricle that is hypertrophied and enlarged. This may cause flow-demand mismatch $(23,24)$. Myocardial ischemia may also be related to increased ventricular wall stress from high afterload (25) and coronary anomalies in transposition of the great vessels (26). Myocardial fibrosis has been demonstrated by cardiac magnetic resonance imaging in patients with Tetralogy of Fallot (27), Eisenmenger Syndrome (28), staged univentricular palliation (29), and those with a systemic right ventricle (30). As a consequence, these lesions are prone to the development of late arrhythmias and systolic dysfunction. The causative mechanism of myocardial fibrosis development is believed 
to be myofibroblast activation and subsequent collagen production (15).

Chronic cyanosis, pulmonary vascular disease, allosensitization, multiple previous operations, and the potential for renal and hepatic failure in Fontan patients (31) can present challenges when ACHD patients develop end stage heart failure (16). Protein losing enteropathy (PLE) is also a significant challenge in patients with failed Fontan physiology (32). It can persist for months after transplant and complicate recovery significantly. Chronic cyanosis can be associated with systemic to pulmonary artery collateral vessels that can cause life-threatening hemorrhage at the time of transplant as well as a volume load to the heart. Pulmonary arteriovenous malformations (AVM) are also commonly seen but can be treated with pre-transplant vascular coils when not diffuse $(16,33)$. AVMs that persist post-transplant may lead to ongoing cyanosis (16). Elevation of pulmonary vascular resistance (PVR) is also important to know prior to listing because it may put the right ventricle at risk of failure post-transplant. A PVR that exceeds 4 Wood units has been associated with increased transplant mortality in ACHD patients (34). However, assessment of PVR can be challenging in the Fontan physiology where it may be assumed to be low. Because of the challenges in assessing PVR prior to organ implantation, varying degrees of right ventricular dysfunction posttransplant should be anticipated. Ruling out secondary causes of elevated PVR such as left heart obstructive lesions is also important because there may be a surgical solution prior to consideration of cardiac transplantation. Univentricular palliation, especially with the neonatal Norwood procedure, is additionally associated with a high incidence of allosensitization (35). Panel reactive antibody levels of greater than $10 \%$ have been associated with increased mortality and can be managed with pre-transplant desensitization protocols (36). The potential causes of heart failure in patients with congenital heart disease are summarized as follows (15):

* Volume overload resulting from left-to-right shunt lesions and valvular regurgitation;

* Pressure overload resulting from valvular disease and other obstructive lesions;

* Ventricular failure related to intrinsic myocardial dysfunction;

* Pulmonary hypertension caused by CHD lesions, ventricular dysfunction, or comorbidities such as obstructive sleep apnea;

* Systemic arterial hypertension resulting from coarctation, acquired renal disease, essential hypertension, or arteriosclerosis;

* Coronary artery disease related to CHD, atherosclerosis, or comorbidities such as diabetes mellitus;

* Cyanosis;

* Intractable arrhythmias or pacemaker induced heart failure.

\section{Indications for transplant}

Life expectancy is one aspect to consider when determining indications for moving forward with cardiac transplantation in ACHD patients. When the extent of heart failure is such that the 1-year survival rate is less than $80 \%$, some reports suggest this as an indication for moving forward with transplant evaluation $(37,38)$. The American Heart Association published consensus guidelines for consideration of transplantation in ACHD patients $(10,15,38)$. While it is difficult to determine specific prognostic indicators of which patients will do well with a transplant and those who won't, the suggested indications for heart transplant are summarized as follows:

* Stage D heart failure refractory to medical therapy with no benefit from surgical, interventional, or electrophysiological intervention;

* Congenital heart disease patients with associated near-sudden death or life-threatening arrhythmias refractory to all therapeutic modalities;

* Stage C heart failure associated with reactive pulmonary hypertension and a potential risk of developing fixed, irreversible elevation of PVR that could preclude heart transplant in the future;

- Fontan patients with PLE and plastic bronchitis;

* Progressive cyanosis leading to functional decline.

\section{Contraindications for transplantation}

When considering contraindications to transplantation, there are absolute contraindications and relative contraindications that are somewhat institution dependent. Reported "absolute" contraindications include (39-41):

* PVR more than 4 Wood units;

* Transpulmonary gradient greater than $12 \mathrm{mmHg}$;

- Malignancy;

* Infection;

* Non-compliance with medical therapy;

* Drug addiction.

Relative contraindications include (39): 


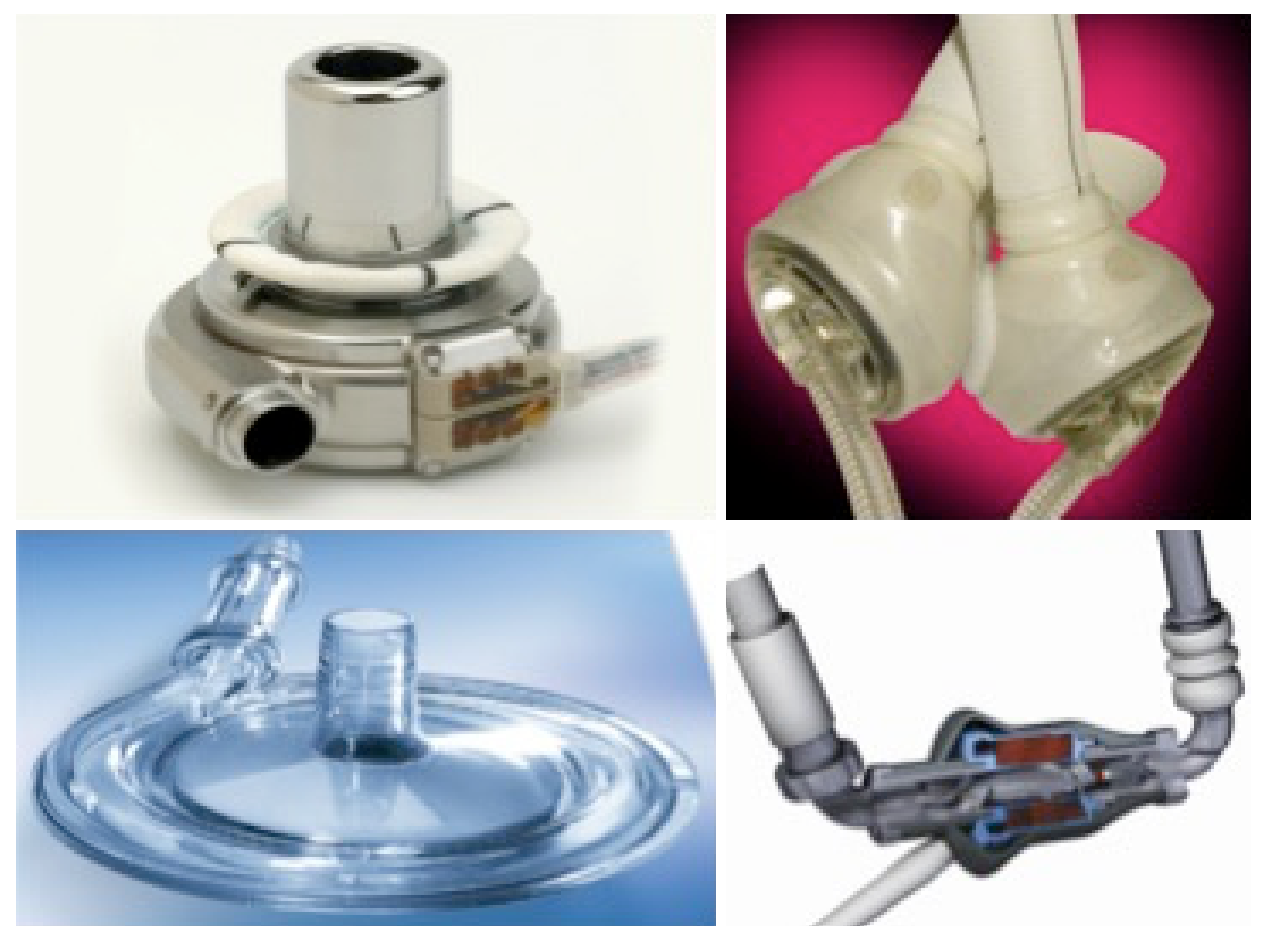

Figure 2 Types of mechanical circulatory support for ACHD patients. ACHD, adult patients with congenital heart disease.

* Obesity $\left(\mathrm{BMI}>30 \mathrm{~kg} / \mathrm{m}^{2}\right)$;

* COPD;

- Severe chronic renal insufficiency (GFR $<30 \mathrm{~mL} / \mathrm{min})$;

* Diabetes with severe peripheral vascular disease;

* Tobacco use.

Nonetheless, the authors do not necessarily agree with some of these recommendations. Our approach is to refer patients with failing Fontan physiology when they become symptomatic from heart failure and/or if they develop signs of end organ dysfunction. Waiting until a Fontan develops PLE or plastic bronchitis is, in our opinion, somewhat late to refer for consideration of cardiac transplantation. Furthermore, a PVR of 4 Wood units is considered a relative rather than an absolute contraindication to transplant, especially with the cadre of medications now available to treat pulmonary vascular disease. It also depends more on the reactivity of the pulmonary vascular bed with provocative testing than the absolute number.

\section{Waiting list and mechanical circulatory support as a bridge to cardiac transplantation}

Attrition for wait-listed heart transplant patients is expected. Clinicians must be vigilant regarding the patient's clinical status and the development of end organ dysfunction. When end organ dysfunction ensues, it may necessitate the use of mechanical circulatory support as a bridge to transplant. In the 2016 ISHLT guidelines of listing criteria for heart transplantation, mechanical circulatory support is recommended when patients develop potentially reversible or treatable comorbidities such as renal failure and pharmacologically reversible pulmonary hypertension (39). A broad selection of devices is available, including extracorporeal membrane oxygenation, pulsatile devices, continuous flow devices, and the total artificial heart (Figure 2). High volume centers, including many pediatric centers, are able to offer these devices across the age spectrum. In 2011, Davies et al. evaluated risk factors for wait-list mortality utilizing the UNOS database over a 15 -year time period from 1995 to 2009 (42). The use of mechanical circulatory support was a risk factor for increased wait-list mortality in ACHD patients (regardless of device type) but not patients without congenital heart disease. In fact, in non-ACHD patients, the use of a ventricular assist device actually lowered wait-list mortality. This data was contradicted by a more contemporaneous report from the International Registry of Mechanically Assisted Circulation (INTERMACS). Vanderpluym and colleagues 
used the INTERMACS registry to evaluate outcomes of circulatory support device use in patients ACHD patients. Over 10 years, there were 126 ACHD patients who received mechanical circulatory support out of 16,182 patients (43). Forty-five of the 126 patients had a systemic morphologic right ventricle. The remaining patients had other forms of UCHD or a morphologic systemic left ventricle. There were several important findings from this study. First, 70\% of patients achieved a positive outcome over 6 months with $21 \%$ successfully bridged to transplant. More than $50 \%$ of patients remained alive on the device. Second, the mortality in the ACHD patients was predominantly in patients undergoing biventricular support or receiving the total artificial heart. These patients were more often INTERMACS class 1 or 2, indicating that they were more high risk at the time of device implant with more end organ dysfunction. Third, ventricular morphology and the presence of UCHD were not predictors of poor outcome. But only 17 of the 126 patients had a UCHD diagnosis (44). The central message of the report was that with proper patient selection, a positive outcome is more often the case when using mechanical circulatory device or support in ACHD patients. With this in mind, others have suggested that it may be time to lower the threshold for use of mechanical circulatory support in this patient population, and that the poor results of the past may be due to the hesitation to place devices in these patients allowing them to become more ill (i.e., INTERMACS 1) (44). Finally, there may be an age advantage when considering mechanical circulatory support in ACHD patients. Rizwan et al. found that the lowest post-transplant survival of bridged congenital heart disease patients was between 15 and 29 years of age. Patients older than 30 years of age that were bridged to transplant, had improved post-transplant outcomes, and the post-transplant survival was comparable to those who were not bridged to transplant with a VAD (45).

\section{Surgical considerations}

The transplant operation for ACHD patients is a challenging anatomic and physiologic undertaking. Patients presenting with previous Fontan reconstruction often require additional surgical procedures at the time of organ implantation. In this population, pulmonary artery, systemic venous, and/or great artery reconstruction are common additional surgical procedures (46). The need for reconstruction of the branch pulmonary arteries needs to be assessed prior to listing. This can be accomplished using cardiac catheterization or CT angiography. The author's approach is to obtain a CT scan for re-operative cases. This aid not only in planning additional surgical reconstructions but is also useful for planning sternal re-entry. Knowing whether or not pulmonary artery reconstruction is necessary may impact the type of donor taken. For example, one may consider avoiding donors where the lungs are being harvested so that the donor pulmonary artery may be used for reconstruction of the recipient pulmonary arteries at the time of organ implantation. The donor pulmonary artery can be used as a free homograft (47) or as a patch. However, lung retrieval is not an absolute contraindication to utilizing a particular donor organ, because the pulmonary arteries can be reconstructed with synthetic patches, though this is not ideal. The need for pulmonary artery reconstruction may also impact the anastomotic sequence during organ implantation. The author's preference is to reconstruct the branch pulmonary arteries prior to the ascending aorta reconstruction. This allows for uninhibited hilum-to-hilum access to the branch pulmonary arteries.

Also notable is that second stage palliation with a hemiFontan can be particularly problematic for pulmonary artery reconstruction and for controlling the large reconstructed neo-aorta in HLHS patients. In this scenario, the branch pulmonary arteries can be very thin walled and densely adherent to the ascending aorta relative to patients palliated with a bidirectional cavopulmonary shunt. For this reason, when faced with transplanting patients palliated with a hemiFontan, the branch pulmonary arteries are not dissected out until establishment of cardiopulmonary bypass. This may help control potentially catastrophic intraoperative bleeding. Finally, because it is difficult to assess PVR pre-implant, we have found right ventricular dysfunction of the implanted graft to be a consistent finding. With that in mind, separating from cardiopulmonary bypass with right heart support is advantageous. We separate from cardiopulmonary bypass with milrinone and inhaled nitric oxide. A subset of ACHD patients, especially those with chronic cyanosis or Fontan physiology, can also be profoundly vasoplegic. In preparation for this, vasopressin and a host of vasoconstrictors should be available to help manage the low SVR state. If while on bypass this state becomes evident, vasopressin is started prior to separating from bypass.

\section{Outcomes}

\section{Survival}

Doumouras and colleagues conducted a meta-analysis of the 


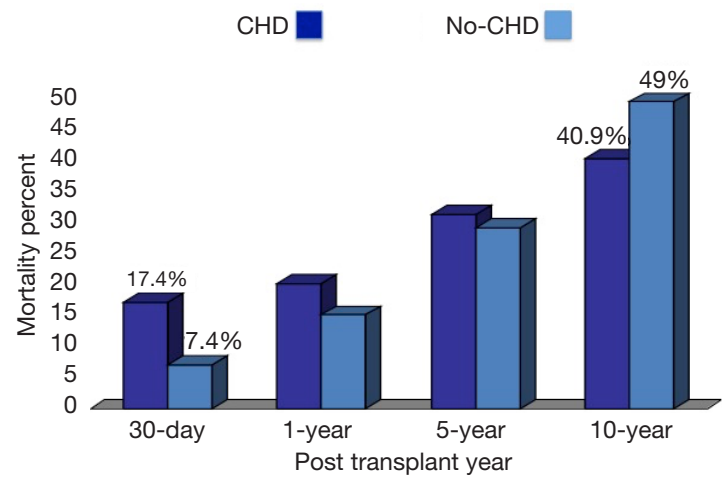

Figure 3 Post-transplant mortality percent at 30-day, 1-, 5-, and 10 -year post-transplant.

available literature on the outcomes of ACHD undergoing cardiac transplantation (48). Mortality was assessed at four different time points: 30-day (operative), 1-, 5-, and 10-year. The survival outcomes in ACHD were compared to a non-CHD cohort of adult patients undergoing heart transplantation. There was a statistically significant difference in survival post-transplant. The 30-day mortality was $17.4 \%$ in ACHD patients versus $7.4 \%$ in non-CHD adult patients undergoing cardiac transplantation. The only other statistically significant survival difference between ACHD and non-CHD adult heart transplant patients was at 10 -year. The mortality was $40.7 \%$ for ACHD patients versus $49.0 \%$ for non-CHD adult patients (48) (Figure 3). Within the ACHD patients undergoing cardiac transplantation, where does the risk lie?

\section{Risk factors for poor outcome post-transplant in ACHD patients}

Several single institution and registry reports have indicated worse post-transplant survival in UCHD patients, particularly Fontan patients. The operative mortality in these reports varied between $14 \%$ to as high as $39 \%$ $(3,5,49,50)$. In later eras, the mortality appeared to be more equivalent between adult patients undergoing cardiac transplantation after the Fontan procedure and non-CHD patients undergoing cardiac transplantation (51). The reported outcomes have been mixed in single institution series. The impact of UCHD was assessed in the metaanalysis by Doumouras and colleagues. The operative mortality in UCHD patients palliated with either a Glenn shunt or total cavopulmonary connection (44\%) was statistically higher than for non-CHD adult patients (14\%) undergoing heart transplantation (48). UCHD patients can include those who have gone through staged palliation or those that remain unpalliated. UCHD patients who did not have a Glenn or Fontan in the Doumouras study had equivalent survival to adult patients undergoing heart transplant without congenital heart disease. This was a novel finding from other reports assessing the impact of UCHD on post-transplant outcome. It suggests that the early mortality after cardiac transplantation for ACHD patients is potentially attributable to palliated UCHD. This is likely related to the complexity of the reconstruction at the time of implant, the high incidence of right ventricular dysfunction of the graft due to persistent elevation of the PVR, and low SVR state from profound vasoplegia.

The Doumouras meta-analysis was limited by the inability to separate out survival outcomes based on Glenn palliation or total cavopulmonary connection since the palliated UCHD patients were analyzed as a group. That analysis was also flawed by the lack of distinction between UCHD patients with impaired or preserved ventricular function. It has been shown that post-Fontan patients with preserved systolic ventricular function have worse post-transplant outcomes $(4,31)$. This finding is related to high incidence of PLE potentially as a result of chronic ventricular volume overload from aortopulmonary collaterals $(52,53)$. Many of these patients also have diastolic dysfunction, and the transplanted heart must tackle the long-term ramifications of diastolic dysfunction (i.e., PVR, venous congestion). Another limitation of the Doumouras meta-analysis is that it did not assess posttransplant survival based on immunosuppression strategy. Karamlou and colleagues conducted an extensive review of cardiac transplantation in ACHD patients using the UNOS database from 1990 to 2008 (14). The use of any induction therapy $(\mathrm{P}=0.008)$ and steroid maintenance therapy were associated with improved survival in all heart recipients. ACHD patients, in particular, gained a survival benefit with maintenance steroids. This finding was consistent with a series of ACHD transplantation reported from the Mayo clinic (1).

\section{Summary}

Experience with cardiac transplantation for ACHD patients with end-stage heart failure is growing. High-risk patient subsets have been defined. Strides have been made in developing evidence-based guidelines of indications for transplant, and the intraoperative management of 
complex reconstruction has evolved. With proper patient selection, a judicious but more aggressive use of mechanical support, and earlier referral of patients with failing Fontan physiology and other complex congenital heart disease, outcomes should continue to improve.

\section{Acknowledgements}

None.

\section{Footnote}

Conflicts of Interest: The authors have no conflicts of interest to declare.

\section{References}

1. Speziali G, Driscoll DJ, Danielson GK, et al. Cardiac transplantation for end-stage congenital heart defects: the Mayo Clinic experience. Mayo Cardiothoracic Transplant Team. Mayo Clin Proc 1998;73:923-8.

2. Thorne S, Deanfield J. Long-term outlook in treated congenital heart disease. Arch Dis Child 1996;75:6-8.

3. Pundi KN, Pundi K, Driscoll DJ, et al. Heart transplantation after Fontan: Results from a surgical Fontan cohort. Pediatr Transplant 2016;20:1087-92.

4. Miller JR, Simpson KE, Epstein DJ, et al. Improved survival after heart transplant for failed Fontan patients with preserved ventricular function. J Heart Lung Transplant 2016;35:877-83.

5. Jayakumar KA, Addonizio LJ, Kichuk-Chrisant MR, et al. Cardiac transplantation after the Fontan or Glenn procedure. J Am Coll Cardiol 2004;44:2065-72.

6. Dipchand AI, Rossano JW, Edwards LB, et al. The Registry of the International Society for Heart and Lung Transplantation: Eighteenth Official Pediatric Heart Transplantation Report--2015; Focus Theme: Early Graft Failure. J Heart Lung Transplant 2015;34:1233-43.

7. Goldfarb SB, Benden C, Edwards LB, et al. The Registry of the International Society for Heart and Lung Transplantation: Eighteenth Official Pediatric Lung and Heart-Lung Transplantation Report--2015; Focus Theme: Early Graft Failure. J Heart Lung Transplant 2015;34:1255-63.

8. Crumb SR, Cook SC, Cheatham JP, et al. Quality outcomes of ACHD patients undergoing cardiovascular procedures and hospital admissions in a free-standing children's hospital. Int J Cardiol 2011;146:326-9.
9. Yap SC, Harris L, Chauhan VS, et al. Identifying high risk in adults with congenital heart disease and atrial arrhythmias. Am J Cardiol 2011;108:723-8.

10. Burchill LJ, Ross HJ. Heart transplantation in adults with end-stage congenital heart disease. Future Cardiol 2012;8:329-42.

11. Diller GP, Kempny A, Alonso-Gonzalez R, et al. Survival prospects and circumstances of death in contemporary adult congenital heart disease patients under follow-up at a large tertiary centre. Circulation 2015;132:2118-25.

12. Verheugt CL, Uiterwaal CS, van der Velde ET, et al. Mortality in adult congenital heart disease. Eur Heart J 2010;31:1220-9.

13. Lund LH, Edwards LB, Kucheryavaya AY, et al. The Registry of the International Society for Heart and Lung Transplantation: Thirty-second Official Adult Heart Transplantation Report--2015; Focus Theme: Early Graft Failure. J Heart Lung Transplant 2015;34:1244-54.

14. Karamlou T, Hirsch J, Welke K, et al. A United Network for Organ Sharing analysis of heart transplantation in adults with congenital heart disease: outcomes and factors associated with mortality and retransplantation. J Thorac Cardiovasc Surg 2010;140:161-8.

15. Stout KK, Broberg CS, Book WM, et al. Chronic Heart Failure in Congenital Heart Disease: A Scientific Statement From the American Heart Association. Circulation 2016;133:770-801.

16. Houyel L, To-Dumortier NT, Lepers Y, et al. Heart transplantation in adults with congenital heart disease. Arch Cardiovasc Dis 2017;110:346-53.

17. Sanchez-Quintana D, Climent V, Ho SY, et al. Myoarchitecture and connective tissue in hearts with tricuspid atresia. Heart 1999;81:182-91.

18. Sedmera D. Form follows function: developmental and physiological view on ventricular myocardial architecture. Eur J Cardiothorac Surg 2005;28:526-8.

19. Tobita K, Keller BB. Right and left ventricular wall deformation patterns in normal and left heart hypoplasia chick embryos. Am J Physiol Heart Circ Physiol 2000;279:H959-69.

20. Rychter Z, Ostadal B. Fate of "sinusoidal" intertrabecular spaces of the cardiac wall after development of the coronary vascular bed in chick embryo. Folia Morphol (Praha) 1971;19:31-44.

21. Stahli BE, Gebhard C, Biaggi P, et al. Left ventricular non-compaction: prevalence in congenital heart disease. Int J Cardiol 2013;167:2477-81.

22. Wessels A, Sedmera D. Developmental anatomy of 
the heart: a tale of mice and man. Physiol Genomics 2003;15:165-76.

23. Hauser M, Bengel FM, Hager A, et al. Impaired myocardial blood flow and coronary flow reserve of the anatomical right systemic ventricle in patients with congenitally corrected transposition of the great arteries. Heart 2003;89:1231-5.

24. Rutz T, de Marchi SF, Schwerzmann M, et al. Right ventricular absolute myocardial blood flow in complex congenital heart disease. Heart 2010;96:1056-62.

25. Sugishita Y, Iida K, Ohtsuka S, et al. Ventricular wall stress revisited. A keystone of cardiology. Jpn Heart J 1994;35:577-87.

26. Ou P, Khraiche D, Celermajer DS, et al. Mechanisms of coronary complications after the arterial switch for transposition of the great arteries. J Thorac Cardiovasc Surg 2013;145:1263-9.

27. Babu-Narayan SV, Kilner PJ, Li W, et al. Ventricular fibrosis suggested by cardiovascular magnetic resonance in adults with repaired tetralogy of fallot and its relationship to adverse markers of clinical outcome. Circulation 2006;113:405-13.

28. Broberg CS, Prasad SK, Carr C, et al. Myocardial fibrosis in Eisenmenger syndrome: a descriptive cohort study exploring associations of late gadolinium enhancement with clinical status and survival. J Cardiovasc Magn Reson 2014;16:32.

29. Broberg CS, Chugh SS, Conklin C, et al. Quantification of diffuse myocardial fibrosis and its association with myocardial dysfunction in congenital heart disease. Circ Cardiovasc Imaging 2010;3:727-34.

30. Babu-Narayan SV, Goktekin O, Moon JC, et al. Late gadolinium enhancement cardiovascular magnetic resonance of the systemic right ventricle in adults with previous atrial redirection surgery for transposition of the great arteries. Circulation 2005;111:2091-8.

31. Simpson KE, Cibulka N, Lee CK, et al. Failed Fontan heart transplant candidates with preserved vs impaired ventricular ejection: 2 distinct patient populations. J Heart Lung Transplant 2012;31:545-7.

32. Backer CL, Russell HM, Pahl E, et al. Heart transplantation for the failing Fontan. Ann Thorac Surg 2013;96:1413-9.

33. Dimopoulos K, Diller GP, Koltsida E, et al. Prevalence, predictors, and prognostic value of renal dysfunction in adults with congenital heart disease. Circulation 2008;117:2320-8.

34. van Riel AC, Schuuring MJ, van Hessen ID, et al.
Contemporary prevalence of pulmonary arterial hypertension in adult congenital heart disease following the updated clinical classification. Int J Cardiol 2014;174:299-305.

35. Meyer SR, Campbell PM, Rutledge JM, et al. Use of an allograft patch in repair of hypoplastic left heart syndrome may complicate future transplantation. Eur J Cardiothorac Surg 2005;27:554-60.

36. Nwakanma LU, Williams JA, Weiss ES, et al. Influence of pretransplant panel-reactive antibody on outcomes in 8,160 heart transplant recipients in recent era. Ann Thorac Surg 2007;84:1556-62; discussion 62-3.

37. Burchill LJ. Heart transplantation in adult congenital heart disease. Heart 2016;102:1871-7.

38. Ross HJ, Law Y, Book WM, et al. Transplantation and Mechanical Circulatory Support in Congenital Heart Disease: A Scientific Statement From the American Heart Association. Circulation 2016;133:802-20.

39. Mehra MR, Canter CE, Hannan MM, et al. The 2016 International Society for Heart Lung Transplantation listing criteria for heart transplantation: A 10-year update. J Heart Lung Transplant 2016;35:1-23.

40. Sian Pincott E, Burch M. Indications for heart transplantation in congenital heart disease. Curr Cardiol Rev 2011;7:51-8.

41. Hascoet S, Baruteau AE, Humbert M, et al. Long-term outcomes of pulmonary arterial hypertension under specific drug therapy in Eisenmenger syndrome. J Heart Lung Transplant 2017;36:386-98.

42. Davies RR, Russo MJ, Yang J, et al. Listing and transplanting adults with congenital heart disease. Circulation 2011;123:759-67.

43. VanderPluym CJ, Cedars A, Eghtesady P, et al. Outcomes following implantation of mechanical circulatory support in adults with congenital heart disease: An analysis of the Interagency Registry for Mechanically Assisted Circulatory Support (INTERMACS). J Heart Lung Transplant 2018;37:89-99.

44. Bryant R 3rd, Morales DLS. Regarding mechanical circulatory support in adults with congenital heart disease: Is it time to lower the threshold for use? J Heart Lung Transplant 2018;37:141-2.

45. Rizwan R, Bryant RR, Zafar F, et al. Inferior transplant outcomes of adolescents and young adults bridged with ventricular assist device. ASAIO J 2017. [Epub ahead of print].

46. Mauchley DC, Mitchell MB. Transplantation in the Fontan patient. Semin Thorac Cardiovasc Surg Pediatr 
Card Surg Annu 2015;18:7-16.

47. Brink J, Saxena P, Yong MS, et al. Pulmonary Artery Reconstruction with Donor Aortic Homograft During Cardiac Transplantation in the Failed Fontan Circulation. J Card Surg 2016;31:65-8.

48. Doumouras BS, Alba AC, Foroutan F, et al. Outcomes in adult congenital heart disease patients undergoing heart transplantation: A systematic review and meta-analysis. J Heart Lung Transplant 2016;35:1337-47.

49. Gamba A, Merlo M, Fiocchi R, et al. Heart transplantation in patients with previous Fontan operations. J Thorac Cardiovasc Surg 2004;127:555-62.

50. Griffiths ER, Kaza AK, Wyler von Ballmoos MC, et al. Evaluating failing Fontans for heart transplantation:

Cite this article as: Bryant $\mathrm{R}$ 3rd, Morales D. Overview of adult congenital heart transplants. Ann Cardiothorac Surg 2018;7(1):143-151. doi: 10.21037/acs.2018.01.01 predictors of death. Ann Thorac Surg 2009;88:558-63; discussion 63-4.

51. Davies RR, Sorabella RA, Yang J, et al. Outcomes after transplantation for "failed" Fontan: a single-institution experience. J Thorac Cardiovasc Surg 2012;143:1183-92.e4.

52. Murtuza B, Hermuzi A, Crossland DS, et al. Impact of mode of failure and end-organ dysfunction on the survival of adult Fontan patients undergoing cardiac transplantation. Eur J Cardiothorac Surg 2017;5 1:135-41.

53. Matsuda H, Ichikawa H, Ueno T, et al. Heart transplantation for adults with congenital heart disease: current status and future prospects. Gen Thorac Cardiovasc Surg 2017;65:309-20. 